Urachal carcinoma is a rare cancer that accounts for $0.5 \%-2 \%$ of all bladder cancers. These tumours are commonly seen in patients 40-70 years of age, twothirds of whom are men. Most urachal cancers are adenocarcinomas but other histological subtypes are described. Patients with urachal cancer may have no symptoms until the late stage. The most common clinical signs are suprapubic mass, haematuria and dysuria. The recommended treatment is primarily surgical, with extended partial cystectomy, en bloc excision of the urachal mass, urachal tract and umbilicus, and pelvic lymph node dissection. While some have advocated radical cystectomy as definitive therapy, this procedure can usually be reserved for larger tumours. Radiation and chemotherapy are ineffective against urachal carcinoma.

The aim of this article is to present a patient with urachal carcinoma and describe the treatment, including the special role of PET-CT.

Key words: carcinoma, urachus, PET-CT.

\section{Carcinoma of the urachus and the role of PET-CT in disease recurrence - case report}

\author{
Marta Biedka ${ }^{12}$, Ewa Ziółkowska', Bogdan Małkowski ${ }^{3}$, Roman Makarewicz
}

1Oddział Radioterapii 1, Centrum Onkologii w Bydgoszczy

2Katedra Onkologii i Klinika Brachyterapii UMK w Toruniu, Collegium Medicum

w Bydgoszczy

3Zakład Medycyny Nuklearnej, Centrum Onkologii w Bydgoszczy

\section{Introduction}

The estimated incidence of urachal carcinoma in the general population is reported to be 1 in 5 million individuals. Most frequently it concerns regions of endemic Schistosoma haematobium infection [1], with a peak incidence at 40-70 years of age, two thirds of patients being men [2]. It is exceedingly rare, with a reported incidence of $0.5 \%$ to $2 \%$ of all bladder tumours. Urachal carcinoma may arise from any of the segments of persisting urachal remnants and they manifest with no clinical symptoms for a long time. The urachus is formed around the 9th week of gestational age, adjacent to the base of the bladder, lies between the umbilical ligaments and extends to the region of the umbilicus, measuring $3-10 \mathrm{~cm}$ in length and $8-10 \mathrm{~mm}$ in diameter. It normally involutes at about the 32nd week of gestational age, ultimately becoming a fibrous cord. Urachal remnants result in various anomalies both in children and adults [2]. Histopathologically, most urachal carcinomas are adenocarcinomas (accounting for 90\%), originating from mucosal cells of the organ, similar to those found in colorectal cancer. In $75 \%$ of cases cancer cells are observed to be mucus-secreting [1]. In most cases cancer infiltration spreads along the bladder wall and patients suffer from abdominal pain, haematuria and dysuria. On physical examination a tumour palpable above the pubic symphysis is observed. In 50-70\% of cases calcifications are found on ultrasound and in CT scanning of the abdomen.

Over the last years the use of positron emission tomography - computed tomography (PET-CT) in various diseases has been evaluated. Completing standard examinations with PET-CT allows more accurate determination of the cancer stage and may result in a change of treatment [4-6]. Up to the present there have been no data on the possible practical use of PET-CT in diagnosing urachal cancer. Equally interesting is its use in the detection of disease recurrence and/or confirmation of distant metastases.

In clinical practice several types of disease stage classification are used. One of the most widely used is the system developed by Sheldon and Ontario (Table I, II). It is assumed that stage T1-T3 patients may potentially be cured. In patients with advanced disease (T4) it is not possible, even with very aggressive treatment [7]. The recommended treatment is major surgery - wide en bloc resection of the tumour with the urachus and the adjacent organs: the bladder, bilateral pelvic lymph nodes, brown tissue, surrounding ligaments, the sigmoid, the umbilicus and part of the anterior abdominal wall [1]. It is a wide, mutilating surgery, hard to accept by the patients. Moreover, in 1993 Henly et al. demonstrated that survival rates were not different between the group with partial resection of the bladder and the patients who underwent radical cystectomy, as long as the surgical margins were tumour free [8]. That is why the approach worldwide is to perform minimally invasive surgery such as laparoscopic removal of the tumour, the bladder dome, the peritoneum, the umbilical ligaments and the umbilicus, but only in cases of locally advanced cancer. 
Table 1. Staging system and outcome of patients with urachal adenocarcinoma

\begin{tabular}{ll} 
Sheldon et al. (1994) & Nakanishi et al. (1996) \\
\hline I no invasion beyond urethral mucosa & \\
\hline II invasion confined to urachus & A invading into the bladder but not abdominal wall, \\
\hline III A local extension into the bladder & peritoneum, or other viscera \\
\hline III B local invasion into abdominal wall & B invading abdominal wall, peritoneum or viscera other than bladder \\
\hline III C local invasion into peritoneum & 42 \\
\hline III D local invasion into viscera other than the bladder & C metastasis to regional lymph nodes or distant sites \\
\hline IV A metastasis to regional lymph nodes & \\
\hline IV B metastasis to distant sites &
\end{tabular}

Table 2. Ontario staging system

$\begin{array}{ll}\text { T } & \\ \text { T1 } & \text { tumour invasion confined to urachus } \\ \text { T2 local extension into the bladder } \\ \text { T3 local invasion to tella adiposa } \\ \text { T4 invasion into peritoneum, abdominal wall, and other viscera }\end{array}$

The attempts of combining surgery with radiotherapy do not deliver the anticipated results. Similarly, minimal or no benefit has been reported for systemic therapy. Currently there is no chemotherapy regimen proven effective for urachal carcinoma. In case of distant metastases the applied systemic therapy gives median survival time of 20 months [1,9].

As the disease predominantly concerns young people and in advanced stages the prognosis is bad, there have been attempts to combine administration of cytostatics with radiotherapy or surgical removal of metastatic foci. There are publications in which combination therapy positively influenced the survival rate. On the other hand, the histopathological type occurring in urachal carcinoma seems to be unresponsive to radiation and systemic therapy [10].

The objective of this paper is to present a patient with urachal carcinoma and share insights on the diagnostic and therapeutic procedures which have been followed in his case. Apparently, it is one of the very few studies in which PET has been used for the assessment of disease recurrence.

\section{Case report}

A 27-year-old male diagnosed with urachal carcinoma presented to the Clinic of Radiotherapy at F. Łukaszczyk Oncology Centre in Bydgoszcz in May 2008, to be qualified for irradiation.

The disease started in November 2006 with abdominal pain and haematuria. After the initial diagnostic tests in Szczecin he was diagnosed with bladder cancer in stage T2NxMo. Due to the histopathological diagnosis (Infiltratio carcinomatosa - carcinoma (G3)), he underwent partial cystectomy. The result of the histopathological examination was carcinoma mucosinum. The patient was discharged with a final diagnosis of urachal cancer and recommendation of close monitoring in the urological clinic.

In 2008 a follow-up ultrasound examination detected the presence of hyperplastic change in the postoperative cavity after the previously removed urachus. A partial resection of the bladder wall with the tumour was performed. In the postoperative histopathological examination the patient was diagnosed with cancer recurrence - adenocarcinoma mucinosum in stage T3NOMO. After surgery the patient reported to the Oncology Centre in Bydgoszcz in order to qualify for systemic therapy and radiotherapy. Ultrasound examination of the abdominal cavity revealed a hypoechogenic area with a vague outline sized $30 \times 50 \times 30 \mathrm{~mm}$ in the postoperative scar, suggesting a keloid. After chemotherapeutic consultation the patient began systemic treatment KG (CBDCA 600 mg, 2000 mg Gemzar). Next, after consultation in the Clinic of Radiotherapy, a decision was taken to perform diagnostic tests and possibly qualify the patient for radiotherapy, due to his young age and the previously performed partial resection of the bladder with confirmed relapse.

The follow-up imaging examinations found a lesion measuring $34 \times 53 \times 42 \mathrm{~mm}$ to the right of the bladder, at the height of the common iliac artery. This area modelled the urinary bladder and connected with the postoperative scar at the front. In the postoperative scar a lesion with uneven contours, measuring $30 \times 30 \mathrm{~mm}$, was depicted. The whole image might have corresponded to tumour recurrence or a thickened postoperative scar. In connection with the vague image in tomography, it was decided to perform positron emission tomography (PET-CT), using labelled fluorodeoxyglucose in order to confirm or exclude active tumour. The PET-CT, after the administration of $600 \mathrm{MBq}$ (18F) 2FDG, revealed a tissue mass measuring $32 \times 59 \times 41 \mathrm{~mm}$, lying between the ilium and the bladder, showing increased radiotracer uptake on the edge of the up to $10 \mathrm{~mm}$ thick lesion, with the exception of the area on the side of the bladder, glucose metabolism evaluated with SUV was 4.1. On the abdominal side the lesion connected with the rectus abdominis and with the thickening of tissue a few centimetres above the pubic symphysis in the subcutaneous tissue measuring $16 \times 31 \times 42 \mathrm{~mm}$; the area showed glucose metabolism SUV of up to 2.9. A similar level of glucose metabolism 


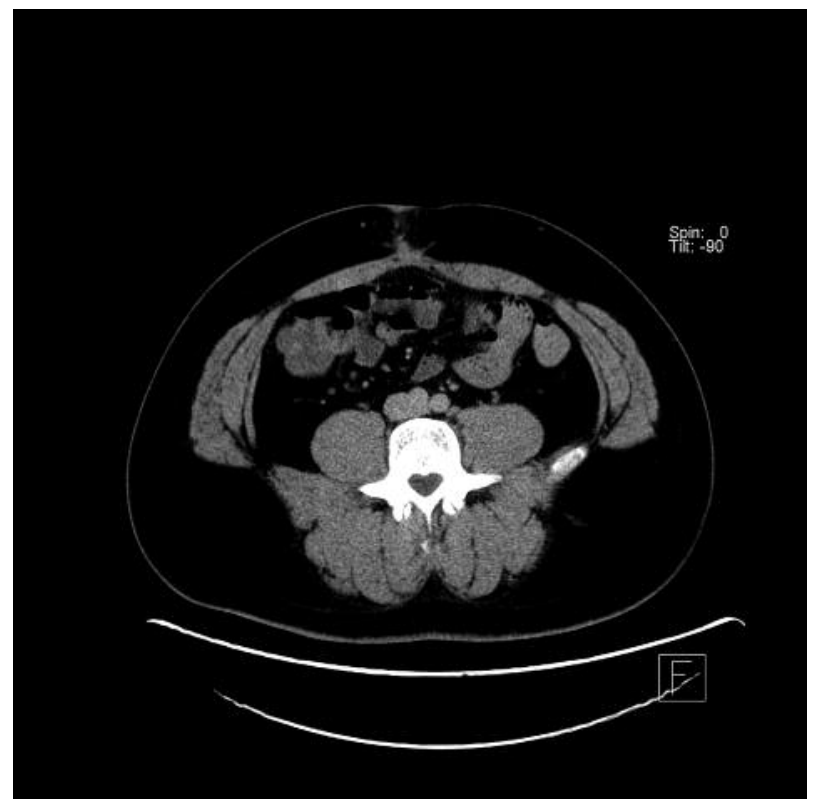

Fig. 1. CT scan of abdomen and pelvis in patient with urachal carcinoma

was visible in the thickening in the subcutaneous layers of tissue, just below the upper pole of the postoperative scar. Moreover, increased radiotracer uptake was observed in seminal vesicles on the right as well as in the prostate gland. The whole image for the clinical interpretation spoke in favour of an active malignancy (Fig. 1, 2, 3). After three courses of chemotherapy the patient was withdrawn from systemic treatment due to disease progression depicted in imaging examinations and PET-CT. Given the patient's young age, the previous treatment for urachal cancer recurrence and a suspicion of another relapse, after the medical consultation the patient underwent surgery. During the surgery the tumour located in the right pelvis was excised along with the lower pole of the scar from the previous surgery. In histopathological examination urachal cancer recurrence was not confirmed (Hp; granulatio et inflammatio, focalis cum fibrosis).

Currently, the patient is feeling well and is under close observation of the Clinic of Radiotherapy at F. Łukaszczyk Oncology Centre in Bydgoszcz. Periodic imaging examinations and urine cytology are performed with no evidence of recurrence.

\section{Discussion}

Urachal cancer is a rare disease; hence the published works represent either small groups of patients or individual cases, on the basis of which it is difficult to draw the proper conclusions. The available literature suggests that major surgery is the best form of treatment. The histological type of urachal cancer seems to be not sensitive to systemic therapy and radiotherapy [10]. In such cases, one should try to resect all tissues and organs derived from the urachus, but such a decision is very difficult for the whole therapeutic team as well as for the patient. The extensive and highly mutilating surgery still arouses great controversy, especially when it concerns young people, forcing the

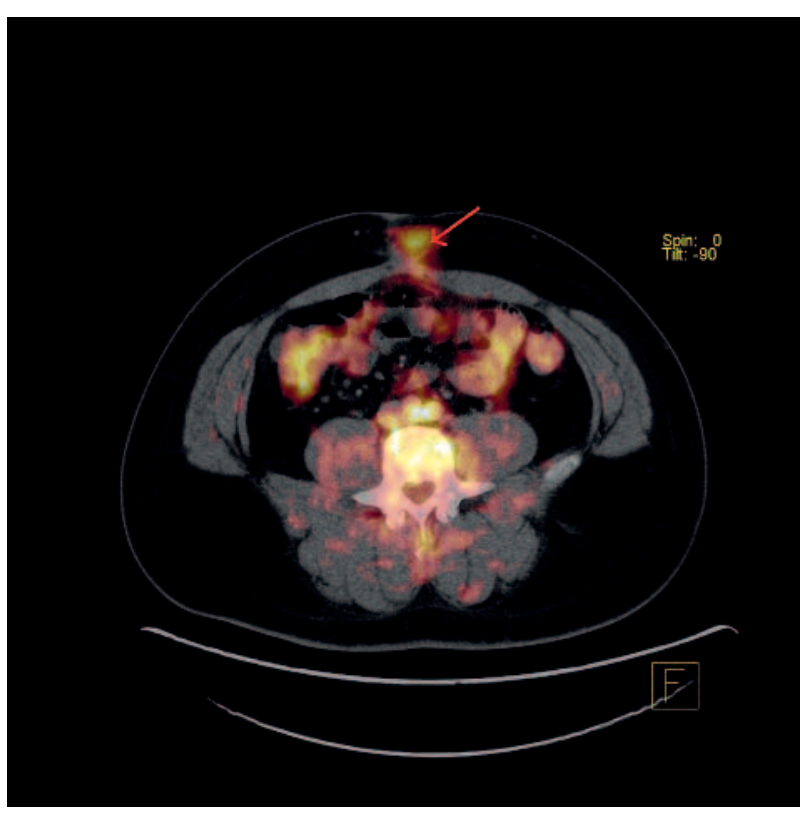

Fig. 2. High uptake of FDG in scar of abdomen

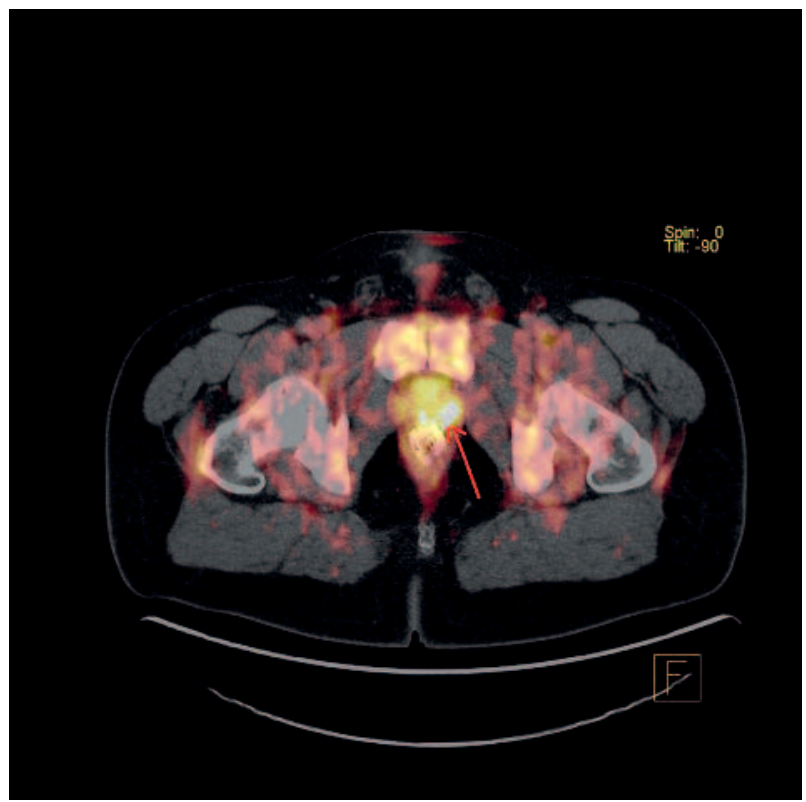

Fig. 3. High uptake of FDG between bladder and iliac bone

team to search for new forms of treatment, which in the case of this disease are doomed to failure. In order to consider the need for invasive treatment in our case it seems necessary to recall that the prognosis for patients with urachal cancer is bad. Disease recurrence is recorded in 51\% of patients after partial cystectomy, and the overall 5-year survival ranges between $11 \%$ and $55 \%$ [1]. Survival is primarily dependent on the stage of the disease, but also on the size of the margin in postoperative histopathological examination (Table I).

No optimal primary treatment in our patient meant the necessity for reoperation, but taking this decision was very difficult. We do not deny that the suspicion of disease recurrence helped qualify the patient for the revision of the 
abdominal cavity. On the one hand we are very pleased that it did not confirm an active neoplastic process. On the other hand, the presented case shows how difficult it might be to correctly interpret the diagnostic tests, which may result in qualifying the patient for unnecessary surgical interventions. In the patient described it was necessary to open the abdominal cavity. Although the bladder was not removed, it is still a very controversial decision, but the scar tissue in which cancer cells may have remained, especially in the area around the bladder and the umbilicus, was excised.

The death rate from recurrent urachal carcinoma is high, even up to $67 \%$; therefore performing radical surgery is of great importance. Herr et al. [7] performed extensive surgery in 50 patients diagnosed with urachal cancer. Survival was $70 \%$, with a median follow-up of 5 years. Worse prognosis is correlated with advancing stages of the disease and metastasis to lymph nodes. In patients with stages I-IIIA 5 -year survival was $93 \%$, falling to $41 \%$ in patients with advanced disease. Survival of patients without lymph node metastases compared to patients with positive nodes was 78 vs. $25 \%$. Distant metastasis was confirmed in $32 \%$ of patients with a mean survival of 22 months from the primary surgery. Median survival after confirmation of metastasis was 17 months. The author emphasizes that the predictors are excision of the urachus and the umbilicus [7].

Urachal carcinoma may metastasize to the liver and the lungs, less frequently to the brain [9]. Kaido et al. [9] presented a patient with urachal cancer, in whom metastasis to the lungs was found and surgically removed 2 years after partial resection of the bladder and chemotherapy. Unfortunately, three years later, MRI revealed a $3 \mathrm{~cm}$ metastasis in the frontal lobe of the brain, and four smaller ones to the left cerebellar hemisphere and left temporal lobe. The biggest lesion was surgically excised, and the others were removed with a Gamma Knife. In MRI, 3 months later, there was evidence of recurrence in the postoperative cavity and 10 postoperative metastatic foci in the entire brain. The surgical excision of the lesion in the brain was repeated. Other foci were treated using a Gamma Knife. Unfortunately, without effect, three months later the patient died. This shows how aggressive this disease is and that we should seek to perform extensive en bloc resection at all costs in order to increase the chances of a cure [9].

The use of PET-CT in urachal cancer seems to be interesting. Performing this test before surgical procedures may help decide on the extent of primary surgery. In the case of our patient such evaluation was not possible, since the patient came to our centre after double surgery. The PET-CT performed 3 months after surgery revealed increased glucose uptake, which might have indicated an active neoplastic process. Based on the result of histopathology, we conclude that the increased uptake of labelled glucose was associated with post-operative scar healing and/or a focus of fibrosis.

In conclusion, we wish to emphasise the significance of the need for a careful preliminary analysis of urachal carcinoma patients and the pivotal role of radical surgery in the successful treatment of this disease. The usefulness of positron emission tomography in assessing clinical stage, diagnosis of local recurrence and/or distant metastases in urachal cancer remains an open issue. Perhaps the lack of baseline PET-CT prior to treatment significantly reduced the usefulness of this test in our patient. It is likely that the use of radiopharmaceuticals other than FDG will have a practical use in this disease.

\section{References}

1. Dahma P, Gschwend J. malignant non-urothelial neoplasms of the urinary bladder: a review. Eur Urol 2003; 44: 672-81.

2. Oyar O, Yesildag A, Gulsoy U, Perk H. The image of urachus adenocarcinoma on Doppler ultrasonography. Eur J Radiol 2002; 44: 48-51.

3. Yeats $M$, Pinch L. Patent urachus with bladder eversion. J Pediatr Surg 2003; 38: 11-3.

4. Herr H, Bochner B, Sharp D, Dalbagni G, Reuter V. Urachal carcinoma: contemporary surgical outcomes. J Urol 2007; 178: 74-78.

5. Chan ES, Ng CF, Chui KL, Lo KL, Hou SM, Yip SK. Novel approach of laparoscopic transperitoneal en block resection of urachal tumor and umbilectomy with a comparison of various techniques. Laparoendosc Adv Surg Tech A 2009; 19: 423-6.

6. Kaido T, Uemura H, Hirao Y, Uranishi R, Nishi N, Sakaki T. Brain metastases from urachal carcinoma. Journal of Clinical Neuroscience 2003; 10: 703-5.

7. Kawakami S, Kageymaka Y, Yonese J, et al. Successful treatment of metastatic adenocarcinoma of the urachus: report of 2 cases with more than 10-year survival. Urology 2001; 58: 462iv-462vi.

8. Chan E, Ng C, Chui K, et al. Novel approach of laparoscopic transperitoneal en block resection of urachal tumor and umbilectomy with a comparison of various techniques. J Laparoendoscopic Adv Surg Techniques 2009; 19: 423-6.

9. Kaido T, Uemura H, Hirao Y, Uranishi R, Nishi N, Sakaki T. Brain metastases from urachal carcinoma. J Clin Neuroscience 2003; 10: $703-5$.

10. Kawakami S, Kageymaka Y, Yonese J, et al. Successful treatment of metastatic adenocarcinoma of the urachus: report of 2 cases with more than 10-year survival. Urology 2001; 58: 462iv-462vi.

\section{Address for correspondence}

Marta Biedka MD PhD

Oddział Radioterapii I

Centrum Onkologii im. F. Łukaszczyka

ul. I. Romanowskiej 2

85-796 Bydgoszcz

tel. +48523743374

e-mail: martabiedka@tlen.pl 